\title{
Histopathological Effects of 5-Fluorouracil on the Liver of Male Rabbits ( with and without Folic Acid administration )
}

Makarem Hameed Ahmad BDS, MSc (Assist Lect)

\author{
Department of Dental Basic Sciences \\ College of Dentistry, University of Mosul
}



\begin{abstract}
Aims : The objective of this study is to evaluate the effects of 5-fluorouracil (5-FU) on the liver tissue of rabbits, as well as the effect of folic acid on the effectiveness of 5-FU on the rabbit's liver. Materials and Methods: In this study nine adult male rabbits ,aged about 3 months were used and divided into three main groups .Group 1 : served as a control group and received normal saline only.Group 2 : treated by 5 fluorouracil , $10 \mathrm{mg} / \mathrm{kg}$ intraperitonealy twice weekly for 8 weeks. Group 3 : treated by 5-fluorouracil , 10 $\mathrm{mg} / \mathrm{kg}$ intraperitonealy twice weekly for 8 weeks with $5 \mathrm{mg}$ of folic acid orally twice weekly for 8 weeks .The animals were sacrificed and livers were excised and processed for histological study. Results: The liver of the treated group 2 shows disturbance in the architecture of hepatic lobule, swelling and vacuolation of the hepatocytes, with ballooning degeneration and areas of necrosis. Congestion in the portal blood vessels and congestion in the central vein , together with congestion and dilatation of the sinusoids. Infiltration of mononuclear inflammatory cells in the portal area and around the central vein .While liver sections of group 3 show slight disturbance in hepatic cords architecture, hepatocytes showed no swelling and few vacuolation in their cytoplasm ,mononuclear cells infilitration mainly in the portal area and in lesser degree than that found in group 2 , necrosis were not present. Conclusion:Toxic effect of 5-FU was observed on the liver tissue of rabbits, and an effective drugs must be applied with anticancer agents to improve liver function .
\end{abstract}

Key words: 5-fluorouracil , liver, rabbit.

Ahmad MH. Histopathological Effects of 5-Fluorouracil on the Liver of Male Rabbits ( with and without Folic Acid administration )Al-Rafidain Dent J. 2016(1):59-66.

Received: 6/10/2013 Sent to Referees: 29/10/2013

Accepted for Publication: 24/11/2013

\section{INTRODUCTION}

Chemotherapy is the treatment with drugs that kill cancer cells or make them less active, it destroys cancer cells anywhere in the body, although chemotherapy is an effective treatment for many types of cancer, chemotherapy like other cancer treatments often causes side effects, it can also harm healthy cells and affect other 
body organs. 5-Fluorouracil, is the most commonly used chemotherapy drug in the treatment of various cancers, including gastrointestinal cancer , breast cancer ,head and neck cancers. It belonges to the family of drugs called antimetabolites. ${ }^{(1)}$

Despite extensive investigations about their hepatotoxicity ,but there is very limited information about their effects on the structure and ultrastructure of liver cells in vivo $^{\text {(2) }}$

It is well known that 5-FU has the potential effect of hepatotoxicity and nephrotoxicity. ${ }^{(3)}$ Thus folinic acid which is the reduced form of folic acid is widely used to prevent the toxic effects of

5-FU and to strengthen the antineoplastic effect of 5-FU. ${ }^{(4)}$ In this study we demonstrate the effect of 5-FU on the liver of the rabbit using light microscopy, together with the effect of folic acid on the rabbit's liver treated with 5-FU.

\section{MATERIALS AND METHODS}

Nine adult male rabbits were used in the present work, aged about 3 months weighing 0.950-1.640 kg, kept in a standardized animal house condition with room temperature $25 \pm 2$ Co and freely fed ,the animals divided into 3 groups ,3 animals for each group .

Group1 : served as a control group ,were injected intraperitonealy with $1 \mathrm{ml}$ of normal saline twice weekly for 8 weeks .
Group 2 : rabbits of this group were treated with $10 \mathrm{mg} / \mathrm{kg}$ of 5 -FU twice weekly for 8 weeks intraperitonealy .

Group 3 : rabbits of this group were treated both with $10 \mathrm{mg} / \mathrm{kg}$ of $5-\mathrm{FU}$ twice weekly intraperitonealy, and 5mg of folic acid orally , for 8 weeks .

Body weight for all the animals were measured at the beginning of the experiment and at the end .Then after 8 weeks all the animals were sacrificed by ether inhalation anesthesia , dissection of the abdomen was done, livers were excised and small pieces of hepatic parenchyma were taken and fixed in $10 \%$ formalin fixative for 24 hours, then dehydrated in ascending grades of alcohol using $50 \%, 70 \%, 90 \%$ and 2 changes of absolute alcohol respectively with a period of one hour for each. Then cleared by 2 changes of xylene with a period of 1 hour for each, then embedded in 3 changes of 60Co melting point paraffin for 2 hours each , sections were obtained and stained with hematoxyline and eosin (H\&E) for light microscopic examinations .(5)

\section{RESULTS}

Physical and clinical observations :

The results demonstrate that there were increase in the body weight of the animals in group 1 and 3 , and there were decrease in the body weight of the animals in group 2 (Table 1).

Table (1): Body weight (gm) of rabbits before and after treatments

\begin{tabular}{cccc}
\hline $\begin{array}{c}\text { Animal } \\
\text { No. }\end{array}$ & Before treatments & $\begin{array}{c}\text { After } \\
\text { Treatments }\end{array}$ & Note \\
\hline 1 & 950 & 1235 & Control \\
2 & 1320 & 1405 & Group I \\
3 & 1400 & 1525 & Twice dose weekly \\
4 & 1595 & 1580 & 5-FU Group II \\
5 & 1640 & 1535 & \\
6 & 1470 & 1425 & Twice dose weekly \\
7 & 1465 & 1500 & 5-FU \& FA \\
8 & 1460 & 1525 & Group III \\
9 & 1270 & 1380 & \\
\hline
\end{tabular}


One of the animals in this group show marked hair loss , and localized alopecia.

Histological findings :

Group 1:

All sections of liver were taken from rabbits in this group appeared to have
The normal histological structure .The hepatic lobule is roughly hexagonal in shape with centrally located terminal hepatic venule (central vein) Figure (1).

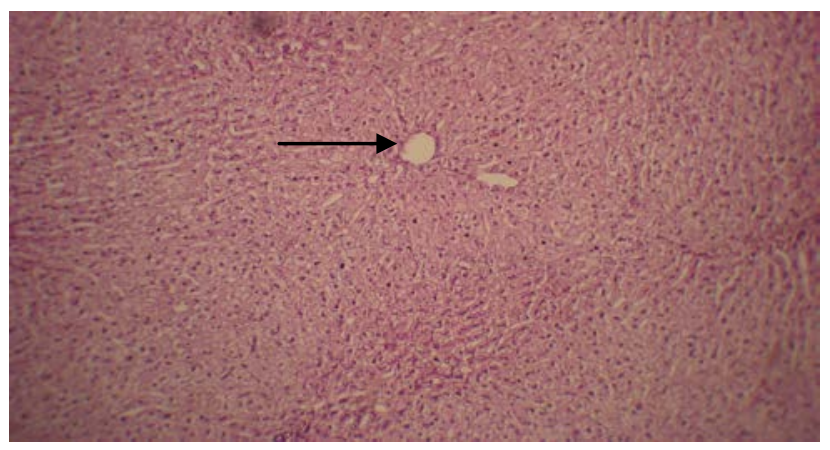

Figure (1) light micrograph of rabbit's liver of group 1 showing the hepatic lobule with it's central vein (arrow).H\&E.( X -90).

The plates of liver cells or hepatocytes and hepatic sinusoids appear to radiate from the central vein to the periphery of hepatic lobule , Figure (2).

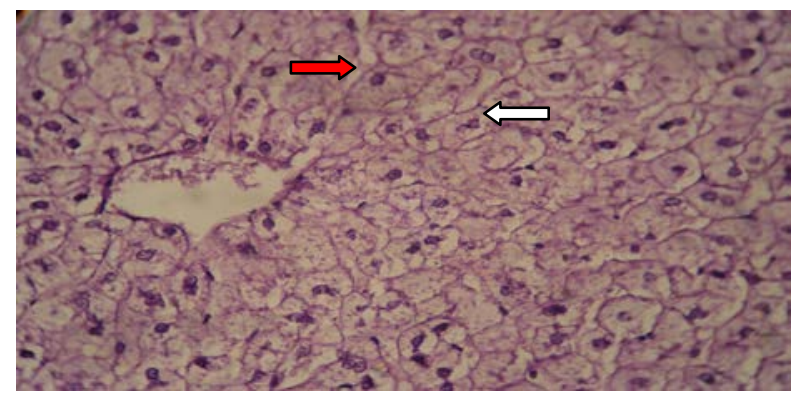

Figure (2) light micrograph of rabbit's liver of group I showing central vein (C.v), hepatocytes (white arrows), sinusoid (red arrow) .H\&E.(X-350)

The portal areas which is located at the corners of the lobules houses the terminal branches of hepatic artery ,portal vein and bile duct . 


\section{Group 2:}

All sections of liver were taken from this group showed similar changes characterized by disturbance in the architecture of hepatic cords, Figure (3). The hepatocytes become swollen and vacuolated Figure (4) giving the appearance of ballooning degeneration with focal areas of necrosis .Congestion in the portal blood vessels, central vein , and sinusoides, Figure (5) . Infilitration of mononuclear inflammatory cells noticed in the portal area, and around the central vein figure (6,7 and 8).

\section{Group 3:}

Liver sections were taken from this group showed slight disturbance in hepatic cords architecture according to that of group 2 . Congestion of blood vessels and sinusoids were not present, but there is dilatation of the sinusoids. Hepatocytes showed neither swelling nor ballooning degeneration ,and the cytoplasmic vacuoles very few Figure (9). Infilitration of mononuclear inflmmatory cells especially in the portal area but in lesser degree than that found in group 2 ,necrosis were not present Figure (10).

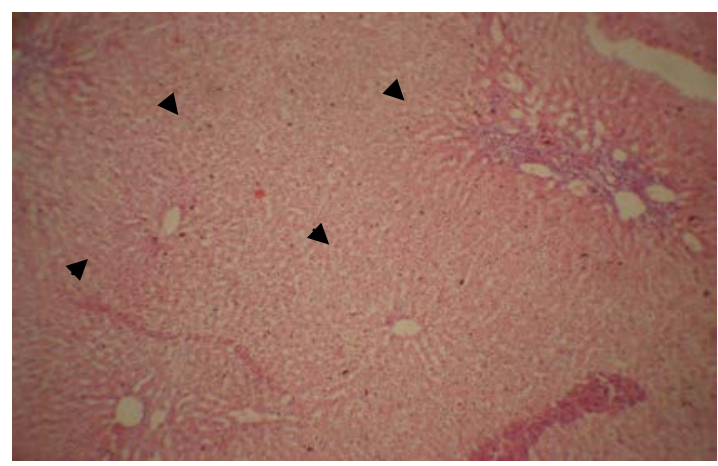

Figure (3): Light micrograph of rabbit's liver of group 2 showing disturbance in the architecture of hepatic cords (arrows).H\&E.(X-75)

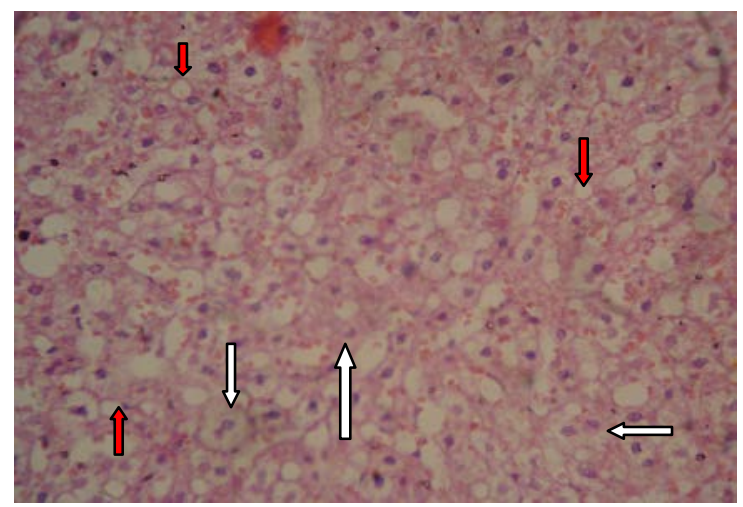

Figure (4): High magnification of figure (3) showing the swelling of hepatocytes (white arrows) ,and the presence of spherical vacuoles in their cytoplasm (red arrows) . H\&E .(X-350). 


\section{5- Fluorouracil and Liver}

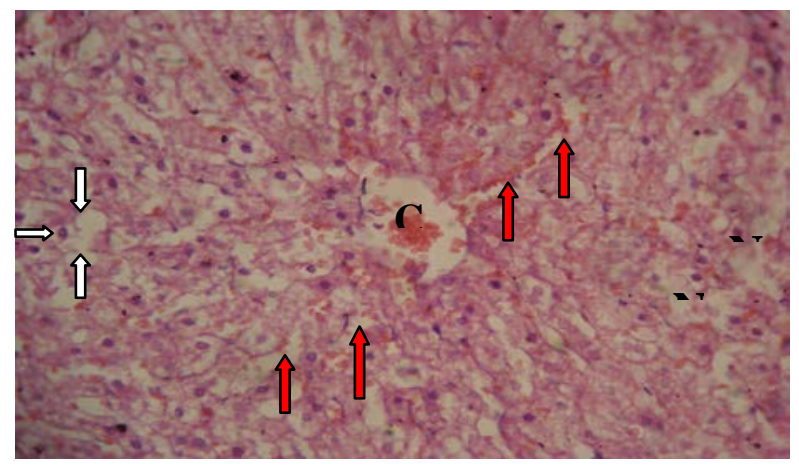

Figure (5): light micrograph of rabbit's liver of group 2 showing congestion of central vein(C.V),ballooning degeneration of hepatocytes (white arrows), sinusoidal dilatation and congestion (red arrows), areas of necrosis (N). H\&E.(X-350)



Figure (6): light micrograph of rabbit's liver of group 2 showing mononuclear cell infilitration in the portal area (arrows). $\mathrm{H} \& \mathrm{E}(\mathrm{X}-75)$

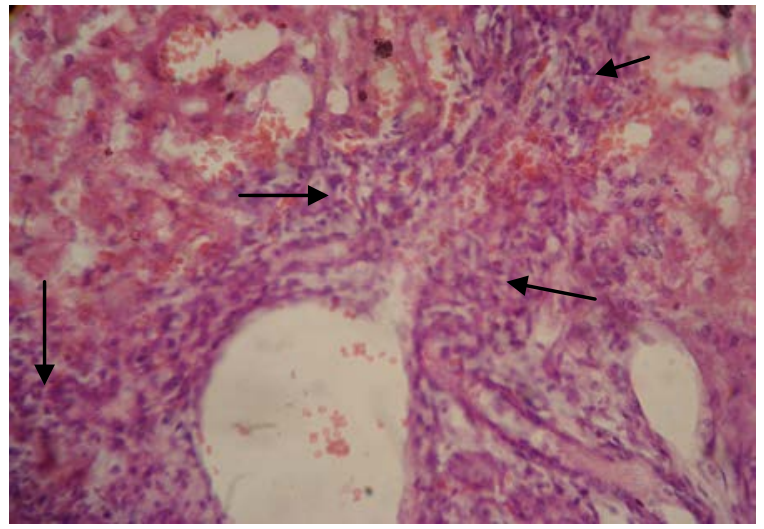

Figure (7): light micrograph of rabbit's liver of group 2 showing mononuclear cell infilitration in the portal area (arrows). H\&E(X-350) 


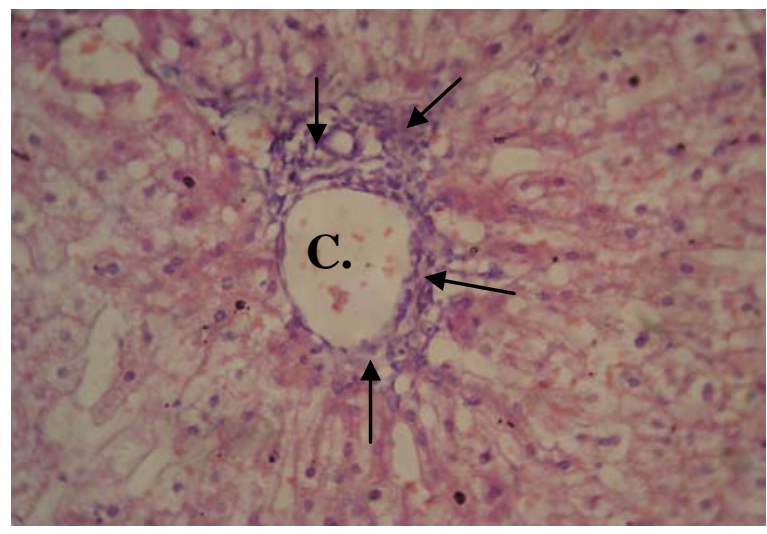

Figure (8): light micrograph of rabbit's liver of group 2 showing mononuclear cell infilitration (arrows) around the central vein(C.V). H\&E(X-350)

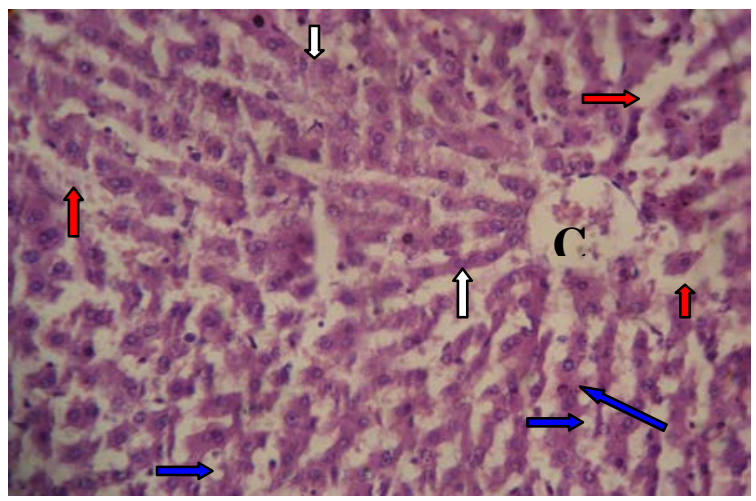

Figure (9): light micrograph of rabbit's liver of group 3 showing hepatic cords (white arrows),central vein (C.V), dilated sinusoids (red arrows), cytoplasmic vacuoles in hepatocytes (blue arrows) . H\&E (X-350)

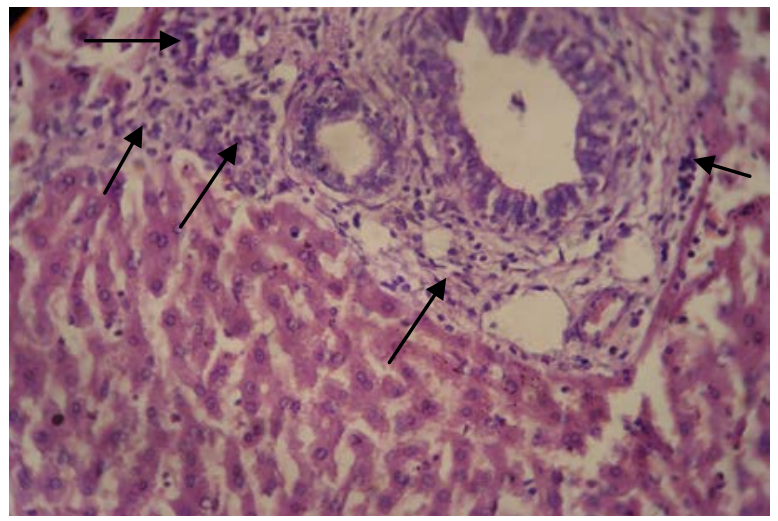

Figure (10): light micrograph of rabbit's liver of group 3 showing mononuclear cell infilitration in and around the portal area (arrows).

H\&E.(X-350). 


\section{DISCUSSION}

Although a number of studies have indicated some side effects of 5-FU as a chemotherapeutic drug of various cancers ,very few studies and limited information about the effect of 5-FU on the structure and ultra structure of liver cells. Various types of chemotherapy-associated hepatopathy have been reported .The effect of Cisplatin , Doxorubicin and 5-FU was demonstrated for the first time on rat liver toxicity using both light and electron microscopy in 2009

(2 )Our study confirms the original finding on the adverse side effects of 5-FU as a chemotherapeutic agent on the rabbit liver. The first histopathological effect seen in group 2 liver is the disturbance in the architecture of hepatic cords and this was mentioned in other studies ${ }^{\text {(6) }}$

The swelling of hepatocytes and the presence of numerous spherical vacuoles in their cytoplasm is mainly consequence of considerable disturbance in lipid droplets and fat metabolism occurring during pathological changes ${ }^{(7)}$ Necrosis was an obvious change seen in the current study is due to that 5FU interferes with the mechanism of action of RNA, resulting from the formation of "fraudulent" RNA by the incorporation of F-UTP (5-fluorouridine-5'-triphosphate), in the ribonucleotides. Incorporation into RNA has been associated with toxicity and has major effects on both the processing and functions of RNA, by interference with the formation of DNA and RNA , 5-FU provokes unbalanced growth and death of cells,(8) and this result is in accordance with other studies which showed many histopathological and ultrastructural abnormalities in the liver after intraperitoneal administration of 5-FU including apoptotic cell death , appearance of numerous areas of inflammatory cells ,and the cytoplasmic organells were markedly affected with collaThe histopathological results of liver sections taken from group 3 revealed that the administration of folic acid has ameliorative effect on the histopathological changes induced by 5-FU , therefore the toxicity of 5FU was significantly greater in folatedeficient rats than in folate supplemented genous fibrils in a number of necrotic cells (2)

Congestion in the portal blood vessels and central vein together with congestion and dilatation of sinusoids are mostly due to inflammation ,after a transient vasoconstriction, vasodilatation occurs resulting in locally increased blood flow and engorgement of the down-stream capillary bed .The microvasculature becomes more permeable resulting in the movement of protein-rich fluid into the extravascular tissue and emigration of leukocytes, this causes RBCs to be more concentrated, thereby increasing blood viscosity and slowing the circulation . These changes are reflected microscopically by numerous dilated small vessels packed with erythrocytes ${ }^{(9)}$ and this effect is in agreements with other studies which demonstrate that non-neoplastic livers exposed to cytotoxic agents showed significantly more frequent sinusoidal dilatation, centrilobular venular fibrosis ,hepatocyte plate disruption ,compared to the control group .(10) However it has been suggested that the initial toxic injury to the sinusoidal endothelial cells by the chemotherapeutic drug may cause sinusoidal wall disruption , activation of hepatic stellate cells and sinusoidal matrix deposition ${ }^{(11)}$ the sinusoids become markedly dilated and red blood cells extravasate into the Disse's spaces through the discontinuities in the endothelial

lining .Infilitration of mononuclear cells (mostly lymphocytes) is a prominent response of body tissues against any injurious agent ${ }^{(8)}$

The decreased in the weight of the animals in group 2 is mostly due to the decreased food intake observed during the period of experiment which is mostly due to the loss of appetite caused by the anticancer therapy . Hepatotoxicity might be contributed to this loss of weight ${ }^{.(12)}$

rats.$^{(13)}$ It has been reported that folic acid has both proliferative and regenerative effects on the liver cells ${ }^{,(14)}$ thus folinic acid which is the reduced form of folic acid is often used to potentialize the antineoplastic effect of 5-FU as a chemical modulator, ${ }^{(4)}$ and there is improvement in liver functions 
and increased bile secretions the day following a chemotherapy protocol including 5-FU and folinic acid in patients with advanced gastric carcinoma . ${ }^{(15)}$

\section{CONCLUSIONS}

Our results confirm that 5-FU cause direct hepatic toxicity, an appropriate protective measures must be applied with anticancer treatment for improving liver function , as a result of our finding we believe that folic acid has more protective effect by preventing the liver hepatotoxicity.

\section{REFERENCES}

1- Longly DB,Harkin DP,Johnston PG. 5Fluorouracil : Mechanisms of action and clinical strategies .Nat Rev Can.2003;3(5):330-8.

2- Hassan I El-Sayyad ,Mohamed F Ismail ,F M Shalaby ,RF Abou-EI- Magd ,Rajiv L Gaur ,Augusta Fernando ,Madhwa HG Raj, Allal Ouhtit. Histopathological effects of cisplatin ,doxorubicin and 5-Fluorouracil on the liver of male albino rats Int J Biol Sci. 2009; 5(5): 466-473.

3- Nora El-Hoseany M.Ali. Protective effect of Captopril against 5- Fluorouracil induced hepato and nephrotoxicity in male albino rats . J Am Sci. 2012;8(2).

4- Bhagavan N V .Nucleotide Metabolism In :Medical Biochemistry. Boston: Jones and Bartlett Publishers . 1992:636-673.

5- Humanson G. Animal tissue technique . SanFrancisco W.H. Freeman and Company 3rd.ed. 1972;125-136.

6- Abdelmeguid NE, Chmaisse NH, Abo Zeinab NS. Silymarin Ameliorates Cisplatin - Induced hepatotoxicity in Rats: and Histopathological Ultrastructural Studies . Pakistan J Biol.Sci.2010;13(10):463-479.
7- Zhang LY.Wang CX. Histopathological and histochemical studies on toxic effect of brodifacoum in mouse liver .Acta Acad Med Sci. 1984 6,(5):386-388.

8- Bertram G.Katzung ,Susan B.Masters , Anthony J.T. Basic and Clinical Pharmacology . 12th ed. The McGraw Hill Companies. 2012;949-970.

9- Kumar V, Cotran RS, Robins LS .Basic Pathology.7th ed. ,Churchill Livingstone ,USA. 2003;33-59.

10- Soo JN, Jai YC, Hye SL, Cheeyoung C, Ja JJ, Yoo SY, Ho SH, and Haeryoung K. Chemotherapy associated hepatopathy in korean colorectal cancer liver metastasis patients :Oxaliplatin-Based Chemotherapy and clinical sinusoidal injury. Korean J Pathol.2012 february 46(1):22-29.

11-Rubbia-Brandt L, Audard V, Sartoretti P, Severe hepatic sinusoidal obstruction associated with oxaliplatin-based chemotherapy in patients with metastatic colorectal cancer. Ann Oncol. 2004;15:460-466.

12-King PD ,Perry MC. Hepatotoxicity of chemotherapy . Oncologist. 2001;6:162-76.

13-Richard F. Branda, Elizabeth Nigels ,Amy R Lafayette and Miles Hacker .Nutritional folate status influences the efficacy and toxicity of chemotherapy in rats. Blood.1998;92:2471-2476.

14-ÖzlemPamukçu Baran, Ayşe Yıldırım, Murat Akkuş.The protective role of folic acid and vitamin E against toxical effects of valproic acid on liver tissue during period of gestation. Dicle T1p Dergisi. 2004;Cilt:31, Sayı:4: 17-23.

15-KajiyamaY, Tsurumaru M, Udagawa $\mathrm{H}$. Relief of jaundice by 5-fluorouracil and folinic acid in patients with recurrent gastric cancer .Surg Oncol. 1996;5:177-181. 